\title{
General Splitting and Merging of 2-D DCT in the DCT Domain
}

\begin{abstract}
Yuh-Jue Chuang, Ting-Jian Pan National Taiwan University, Taipei, Taiwan, R.O.C.

Abstract-An efficient method for splitting an N $\times \mathrm{N}$ 2-D DCT block into four $(\mathrm{N} / 2) \times(\mathrm{N} / 2)$ or two $\mathrm{N} \times(\mathrm{N} / 2)$ (or $(\mathrm{N} / 2) \times \mathrm{N}) 2-\mathrm{D}$ DCT blocks is presented and vice versa. The computational complexity of the proposed methods is lower than the direct approach and the same as the most efficient converting approach existed in the literature. Besides, the proposed DCT splitter/merger is suitable for implementation by specific multimedia instruction set available nowadays. When $\mathrm{N}=8$, our method can be applied to realize the transcoding between the latest video coding standards $\mathrm{AVC} / \mathrm{H.264}$ and the older ones, such as MPEG-1, MPEG-2 and MPEG-4 part 2.
\end{abstract}

Department of Computer Science and Information Engineering Department of Computer Science and Information Engineering

\section{INTRODUCTION}

Multimedia signals, such as images and video, are always compressed so as to save memory space and/or be easily transmitted via network. However, the signals have to be processed before being displayed, transmitted, printed, etc. Some of the frequently used manipulations are scaling, filtering, rotation and translation. Implementing these functions in the compressed domain is advantageous from the computational complexity point of view as well as the image quality and the memory usage. This is because the transition to the time or spatial domain and the recompression of the data are avoided. All existing compression standards-JPEG, MPEG1, MPEG2 and H.26x - are based on the discrete cosine transform (DCT), which is applied to blocks of data of certain lengths. Our work aims to directly split an $N \times N$ 2-D DCT into four adjacent $(N / 2) \times(N / 2)$ 2-D DCTs without the need of performing an $N \times N$ IDCT and the four $(N / 2) \times(N / 2)$ DCTs. On the other hand, merging four adjacent $(N / 2) \times(N / 2) 2$ 2-D DCTs into one $N \times N$ DCT can also be realized by inverse transposing the "split" case. The block diagram of the proposed 1-D DCT splitter/merger is shown in Fig.1(b), where DCT splitter/merger module have to be implemented by using multiplications, additions and permutations as little as possible. We will show the superiority of the proposed algorithm in computational complexity from both the algorithmic-level and the programming-level.

This paper is organized as follows. In Section II, the splitter/merger of the 1-D/2-D DCT is addressed. Two kinds of computational complexity comparisons with other approaches are given in Section III. Finally, Section IV concludes this write-up.

\section{I. THE DCT SPLITTER AND/OR MERGER}

For the sake of simplicity, let us confine our attention to the 1-D case, first. Assume that the DCT coefficients $Y_{k}$ and $Z_{k}, k=0, \ldots, N / 2$, of two consecutive N/2-point data sequences $y=\left[\begin{array}{llll}y_{0} & y_{1} & \cdots & y_{N / 2}\end{array}\right]^{t}$ and $z=\left[\begin{array}{llll}z_{0} & z_{1} & \cdots & z_{N / 2}\end{array}\right]^{t}$ are given. The problem to be addressed is: how to efficiently compute the $N$-point DCT coefficients $X_{k}, k=0,1, \ldots, N$, where $X_{k}$ stands for the DCT coefficients of

$x=\left[\begin{array}{llll}x_{0} & x_{1} & \cdots & x_{N}\end{array}\right]^{t}=\left[\begin{array}{llllllll}y_{0} & y_{1} & \cdots & y_{N / 2} & z_{0} & z_{1} & \cdots & z_{N / 2}\end{array}\right]^{\prime}$.

The normalized forward DCT Type-Il (DCT-II) of a length- $N$ sequence $x_{n}$ is, given in [1], as follows:

$$
X_{k}=\varepsilon_{k} \sum_{n=0}^{N-1} x_{n} \cdot \cos \left(\frac{\pi(2 n+1) k}{2 \cdot N}\right) \text { for } k=0,1, \ldots, N-1
$$

where. $\varepsilon_{k}=\sqrt{\frac{1}{N}}$, for $k=0$ and $\varepsilon_{k}=\sqrt{\frac{2}{N}}$, for $k \neq 0$. The DCT-II's of the length-N/2 sequences $y$ and $z$ can be represented by similar expressions. The DCT defined in (1)

\section{Ja-Ling Wu Senior Member, IEEE}

National Taiwan University, Taipei, Taiwan, R.O.C

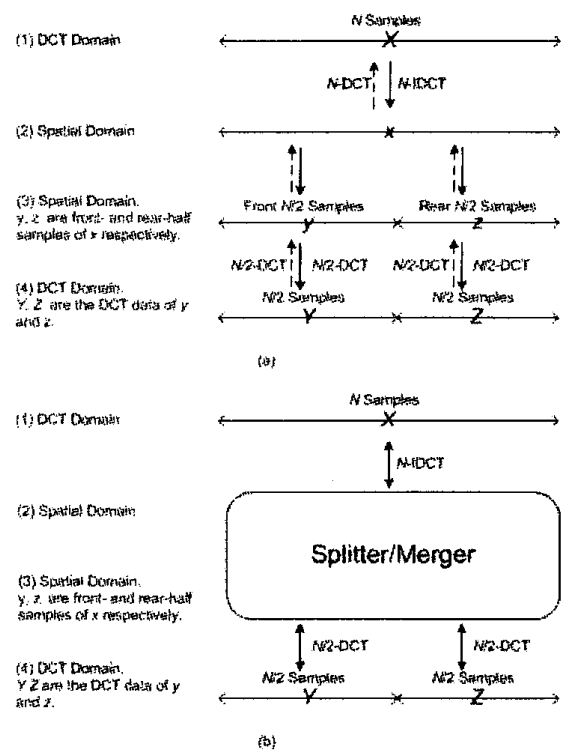

Fig.1. (a) The traditional approach, and (b) The proposed approach for realizing the 1-D DCT splitter/merger.

can be written in matrix form as

$$
X=\left[T_{N}^{\prime \prime}\right] x=\left[C_{N}\right]\left[T_{N}\right] x
$$

where $\left[C_{N}\right]=\operatorname{diag}\left(\varepsilon_{0}, \varepsilon_{l}, \ldots, \varepsilon_{N-1}\right)$ and $\left[T_{N}\right]$ is an $N \times N$ matrix with elements $\left[T_{N}\right]_{i j}=\cos \left(\frac{\pi(2 j+1) i}{2 \cdot N}\right), i, j=0,1, \ldots, N-1$. In transform coding applications, the DCT stage is usually followed by a quantization stage. The computation of $\varepsilon_{k}$ can be saved by absorbing them into the quantization stage (the so-called non-scale case). In this case, we rewrite Eqn.(2) as

$$
X=\left[T_{N}\right] x
$$

A. Use recursive form $\left[\hat{T}_{N}\right]$ instead of $\left[T_{N}\right]$ :

Instead of viewing (3) as the real part of a scaled $N$-point DFT, Hou [2] delved into the symmetry of the kernel in (3) and partitioned the transform matrix $\left[T_{N}\right]$ into a recursive form. Let $\left[\hat{T}_{N}\right]=\left[\widetilde{P}_{N}\right]\left[T_{N}\right]\left[\bar{P}_{N}\right]^{-1}$, it can be examined that

$$
\left[\hat{T}_{N}\right]=\left[\begin{array}{cc}
{\left[\hat{T}_{N / 2}\right]} & {\left[\hat{T}_{N / 2}\right]} \\
{\left[\hat{D}_{N / 2}\right]} & -\left[\hat{D}_{N / 2}\right]
\end{array}\right],
$$

where $\left[\widetilde{P}_{N}\right]$ is an $(N \times N)$ bit-reversal matrix and $\left[\bar{P}_{N}\right]$ is a lso an $(N \times N)$ permutation matrix defined as

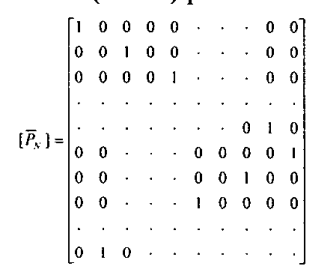

- This work was partially supported by the National Science Council and the Ministry of Education of ROC under the contract No. NSC92-2622-E-002-002.

NSC92-2213-E-002-023 and 89E-FA06-2-4-8. 
To simply our notation, let $\phi_{n}=(n+1 / 4)(2 \pi / N)$. $\left[\hat{D}_{s / 2}\right]$ can be further factorized as

$$
\left[\hat{D}_{N / 2}\right]=\left[K_{N / 2}\right]\left[\hat{T}_{N / 2}\right]\left[Q_{N / 2}\right]
$$

where $\quad\left[Q_{N / 2}\right]=\operatorname{diag}\left\{\cos \left(\phi_{0}\right), \cdots, \cos \left(\phi_{N / 2-1}\right)\right\} . \quad$ and $\left[K_{N / 2}\right]=\left[\widetilde{P}_{N / 2}\right]\left[L_{N / 2}\right]\left[\widetilde{P}_{N / 2}\right]$. In which, $\left[L_{N / 2}\right]$ is the lower $(N / 2) \times$ $(N / 2)$ triangular matrix given by

$$
\left[L_{N / 2}\right]=\left[\begin{array}{cccccc}
1 & 0 & 0 & 0 & \cdots & 0 \\
-1 & 2 & 0 & 0 & \cdots & 0 \\
1 & -2 & 2 & 0 & \cdots & 0 \\
-1 & 2 & -2 & 2 & \cdots & 0 \\
\vdots & \vdots & \vdots & \vdots & \cdots & 0 \\
-1 & 2 & -2 & 2 & \cdots & 2
\end{array}\right]
$$

The formal structure is made clear by following equations:

$$
X=\left[\widetilde{P}_{N}\right]\left[\hat{T}_{*}\right]\left[\bar{P}_{s}\right] x
$$

$$
=\left[\widetilde{P}_{N}\right]\left[\begin{array}{cc}
{\left[\hat{T}_{N / 2}\right]} & {\left[\hat{T}_{N / 2}\right]} \\
{\left[K_{s / 2}\right]\left[\hat{T}_{N / 2}\right]\left[Q_{N / 2}\right]} & -\left[K_{N / 2}\right]\left[\hat{T}_{N / 2}\right]\left[Q_{N / 2}\right]
\end{array}\right]\left[\bar{P}_{x}\right] x
$$

The transform matrix $\left[\hat{T}_{N}\right]$ can be delineated by considering the block matrix factorization as:

$\left[\hat{T}_{N}\right]=\left[\begin{array}{cc}{\left[I_{N / 2}\right]} & 0 \\ 0 & {\left[K_{N / 2}\right]}\end{array}\right]\left[\begin{array}{cc}{\left[\hat{T}_{N / 2}\right]} & 0 \\ 0 & {\left[\hat{T}_{N / 2}\right]}\end{array}\right]\left[\begin{array}{cc}{\left[I_{N / 2}\right]} & 0 \\ 0 & {\left[Q_{N / 2}\right]}\end{array}\right]\left[\begin{array}{cc}{\left[I_{N / 2}\right]} & {\left[I_{N / 2}\right]} \\ {\left[I_{N / 2}\right]} & -\left[I_{s / 2}\right]\end{array}\right](7)$

B. Efficient DCT domain split and merge algorithms

\section{B.1 One dimensional case}

Define $\left[U_{N}\right]$ as another permutation matrix such that $\left[U_{N}\right]\left[\bar{P}_{N}\right] x=\left[\left[\bar{P}_{N / 2}\right] y\right]$. It can be verified that $\left[U_{N}\right]^{-1}=\left[U_{N}\right]^{t}$ and

$$
\left[U_{\mathrm{v}}\right]=\left[\begin{array}{cccc}
\left.I_{x+4}\right] & 0 & 0 & 0 \\
0 & 0 & 0 & {\left[I_{x, 4]}\right]} \\
0 & {\left[U_{x,+1}\right]} & 0 & 0 \\
0 & 0 & {\left[I_{x+1}\right]} & 0
\end{array}\right] .
$$

Substituting (7) and (8) into (6) leads to

$$
X=\left[\tilde{P}_{N 3}\right]\left[\begin{array}{cc}
{\left[I_{N / 2}\right]} & 0 \\
0 & {\left[K_{N / 2}\right]\left[M^{\prime} N_{N / 2}\right]}
\end{array}\right]\left[\begin{array}{l}
{\left[\hat{T}_{N / 2}\right]\left[\bar{P}_{N / 2}\right] y+\left[\hat{T}_{N / 2}\right]\left[\tilde{P}_{N / 2}\right] y-\left[\tilde{T}_{N / 2}\right]\left[\tilde{I}_{N / 2}\right]\left[\bar{P}_{N / 2}\right] z} \\
\left.\bar{P}_{N / 2}\right] z
\end{array}\right] \text {. }
$$

where $\left[M_{N / 2}^{\prime}\right]=\left[\hat{T}_{N / 2}\right]\left[Q_{N / 2}^{\prime}\right]\left[\hat{T}_{N / 2}\right]^{-1}, \quad\left[\tilde{I}_{N / 2}\right]=\left[\begin{array}{cc}0 & {\left[I_{N / 4}\right.} \\ {\left[I_{N / 4}\right]} & 0\end{array}\right]$ and $\left[Q_{N / 2}^{\prime}\right]=\left[Q_{N / 2}\left[\begin{array}{cc}{\left[I_{N / 4}\right]} & 0 \\ 0 & -\left[I_{N / 4}\right]\end{array}\right]\right.$.

Consequently,

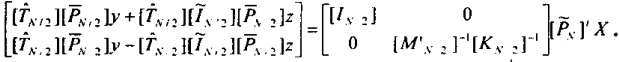

Then, it is clear that

$$
\begin{aligned}
& {\left[\hat{T}_{N / 2}\right]\left[\bar{P}_{s / 2}\right] y=\left(\left[\widetilde{P}_{s / 2}\right] X_{v}+\left[M_{N / 2}^{\prime}\right]^{-1}\left[K_{s / 2}\right]^{-1}\left[\widetilde{P}_{s / 2}\right] X_{o}\right) / 2,} \\
& \text { and } \\
& {\left[\hat{T}_{N / 2}\right]\left[\widetilde{T}_{v / 2}\right]\left[\bar{P}_{v / 2}\right] z=\left(\left[\widetilde{P}_{s / 2}\right] X_{c}-\left[M_{N / 2}^{\prime}\right]^{-1}\left[K_{v / 2}\right]^{-1}\left[\widetilde{P}_{s / 2}\right] X_{o}\right) / 2,}
\end{aligned}
$$

where $\left(X_{e}\right)$ and $\left(X_{o}\right)$ are the even- and odd- numbered transformed components of $X$, and it can be easily to verify

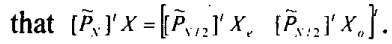

Notice that $\left[\hat{T}_{s / 2}\right]\left[\tilde{P}_{s / 2}\right] y=\left[\widetilde{P}_{s / 2}\right] Y$, as shown in (11), is a reordered version of $Y$, and if we set $Z=\left[J_{v / 2}\right]\left[\hat{T}_{v / 2}\right]\left[\tilde{T}_{s / 2}\right]\left[\bar{P}_{v / 2}\right] z$, then one can show that $\left[J_{N}\right]=\left[\widetilde{P}_{x}\right]\left[\begin{array}{cc}{\left[I_{N / 2}\right]} & 0 \\ 0 & -\left[I_{1 / 2}\right]\end{array}\right]$.

Hence, the splitter algorithm can be described in matrix form as $\left[\begin{array}{l}Y \\ Z\end{array}\right]=\left[S_{x}[X]\right.$ and the merger algorithm is $[X]=\left[S_{N}\right]^{\}^{-}}\left[\begin{array}{l}Y^{*} \\ Z\end{array}\right]$, where

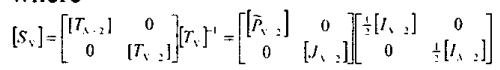

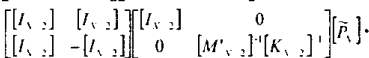

\section{B.2 Two dimensional case}

Let [ $G$ ] be the $N \times N$ two-dimensional (2D) DCT-II of the $N \times N$ data matrix $[g]$, and their relationship can be represented in matrix form as:

$$
[G]=\left[T_{N}\right][g]\left[T_{N}\right]^{-1} \text { and }[g]=\left[T_{N}\right]^{-1}[G]\left[T_{N}\right]
$$

Eqn.(15) implies that the 2D-DCT can be implemented by performing a series of 1-D transforms. The data matrix $[g]$ can be decomposed into four $(N / 2) \times(N / 2)$ data blocks, $\left[g_{l}\right]$, $\left[g_{2}\right],\left[g_{3}\right]$ and $\left[g_{4}\right]$. Our goal is to split $[G]$ into four $(N / 2) \times$ $(N / 2)$ matrices $\left[G_{I}\right],\left[G_{2}\right],\left[G_{3}\right]$ and $\left[G_{4}\right]$, which respectively are the DCTs of $\left[g_{I}\right],\left[g_{2}\right],\left[g_{3}\right]$ and $\left[g_{4}\right]$, without the need of using an $N \times N$-IDCT and four $(N / 2) \times(N / 2)$-DCTs. Follow the same derivation given in subsection II.A, the split algorithm can be written as

$$
\begin{aligned}
{\left[\begin{array}{ll}
{\left[G_{1}\right]} & {\left[G_{2}\right]} \\
{\left[G_{3}\right]} & {\left[G_{4}\right]}
\end{array}\right] } & =\left[\begin{array}{cc}
{\left[T_{N / 2}\right]} & 0 \\
0 & {\left[T_{N / 2}\right]}
\end{array}\right]\left[T_{N}\right]^{-1}\left[G \mathbb{I}_{N}\right]\left[\begin{array}{cc}
{\left[T_{N / 2}\right]^{-1}} & 0 \\
0 & {\left[T_{N / 2}\right]^{-1}}
\end{array}\right], \\
& =\left[S_{*} \mathbb{I} G \mathbb{I} S_{N}\right]^{-1}
\end{aligned}
$$

and the merge algorithm as

$$
[G]=\left[S_{N}\right]^{-1}\left[\begin{array}{ll}
{\left[G_{1}\right]} & {\left[G_{2}\right]} \\
{\left[G_{3}\right]} & {\left[G_{4}\right]}
\end{array}\right]\left[S_{N}\right] .
$$

We can also respectively split $[G]$ into two $N \times(N / 2)$ or $(N / 2)$ $\times N$ DCT blocks as

$$
\left[\begin{array}{ll}
{\left[G_{1}\right.} & G_{2} \\
{\left[G_{3}\right.} & G_{4}
\end{array}\right]=\left[\begin{array}{cc}
{\left[T_{N / 2}\right]} & 0 \\
0 & {\left[T_{N / 2}\right]}
\end{array}\right]\left[T_{N}\right]^{-1}[G]\left[T_{N} \mathbb{I} T_{N}\right]^{-1}=\left[S_{N} \mathbb{I} G\right]
$$

and

$$
\left[\left[\begin{array}{l}
G_{1} \\
G_{3}
\end{array}\right]\left[\begin{array}{l}
G_{2} \\
G_{4}
\end{array}\right]\right]=\left[T_{N} \mathbf{I} T_{N}\right]^{-1}\left[G \mathbb{\llbracket} T_{N}\left[\begin{array}{cc}
{\left[T_{N / 2}\right]^{-1}} & 0 \\
0 & {\left[T_{N / 2}\right]^{-1}}
\end{array}\right]=\left[G \llbracket S_{N}\right]^{-1} .\right.
$$

Notice that, these two kinds of 2-D DCTs had also been included in early versions of the AVC/H.264[3] for $N=8$.

\section{A concrete example: the $(8 \times 8)$ to $(4 \times 4)$ DCT-II splitter}

For a long time, the $8 \times 8$ DCT has been adopted extensively by the major coding standards for representing digital image, delivering broadcast video and making personal visual communications. Nowadays, the latest AVC/H.264 [4] video coding standard provides the capability to predict samples locally down to $4 \times 4$ blocks before operating transform coding. As a result, an impressive compression gain has been obtained, about 1.5 to 2.0 times as compared with the MPEG-4 part 2, while a nearly the same perceptual picture quality will be kept. Due to the wide-spreading of DVD and the trend of HDTV/DTV, most existing video contents are still encoded in MPEG-2 form. Therefore, the MPEG-2-to-AVC/H.264 transcoder or vice versa, is believed to be an indispensable functional module of future video signal treatments.

The simplest way to develop an MPEG-2-to-AVC/H.264 transcoder is directly cascading an $8 \times 8$ IDCT with four $4 \times 4$ DCTs. Intuitively, this direct approach is considered to be computationally intensive and suffers from certain degree of quality loss. On the other hand, by using the DCT splitter and/or merger has discussed in last section, a more efficient and precise video transcoder can be implemented in the DCT transform domain, directly. According to Eqns.(16) and (17), an $8 \times 8$ DCT-II block [G] can be split into four $4 \times 4$ DCT-II blocks $\left[G_{l}\right],\left[G_{2}\right],\left[G_{3}\right]$ and $\left[G_{4}\right]$ and vice versa as

$\left[\begin{array}{ll}{\left[G_{1}\right]} & {\left[G_{2}\right]} \\ {\left[G_{3}\right]} & {\left[G_{4}\right]}\end{array}\right]=\left[S_{8} \llbracket[G]\left[S_{8}\right]^{-1}\right.$ and $[G]=\left[S_{8}\right]^{-1}\left[\begin{array}{ll}{\left[G_{1}\right]} & {\left[G_{2}\right]} \\ {\left[G_{3}\right]} & {\left[G_{4}\right]}\end{array}\right]\left[S_{8}\right]$ where

$\left[S_{8}\right]=\left[\begin{array}{cc}{\left[\widetilde{P}_{4}\right]} & 0 \\ 0 & {\left[J_{4}\right]}\end{array}\right]\left[\begin{array}{cc}\frac{1}{2}\left[I_{4} \rrbracket\right. & 0 \\ 0 & \frac{1}{2}\left[I_{4}\right]\end{array}\right]\left[\begin{array}{cc}{\left[I_{4}\right]} & {\left[I_{4}\right]} \\ {\left[I_{4}\right]} & -\left[I_{4}\right]\end{array}\right]\left[\begin{array}{cc}{\left[I_{4}\right]} & 0 \\ 0 & {\left[M^{\prime}\right]^{-1}\left[K_{4}\right]^{-1}}\end{array}\right]\left[\widetilde{P}_{8}\right] .(21)$

The signal-flow graph for realizing the proposed transform 
domain splitting and merging between one 8-point DCT and two 4-point DCTs is depicted in Fig.2.
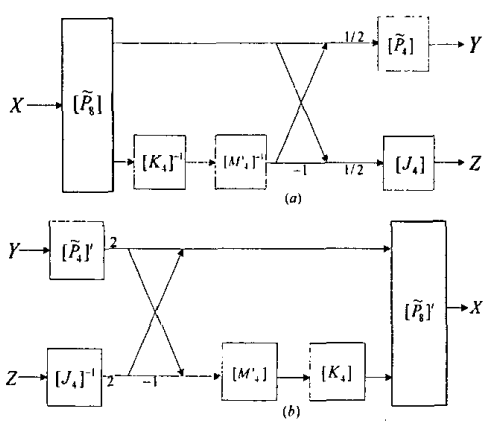

Fig.2. The signal-flow graph for (a) Splitting an 8-point DCT to two 4-point DCTs, and (b) Merging two 4-point DCTs to an 8-point DCT.

\section{COMPUTATIONAL COMPLEXITY ANALYSES}

In this section, two computational complexity analyses will be discussed. First, the algorithmic-level analysis, i.e., the number of multiplications and additions required for the proposed approach will be counted in comparison with other ones in the literature. Nowadays, most of the modern processors have specific multimedia instruction set to facilitate multimedia applications. For example, the single-instruction-multiple-data (SIMD) execution model was introduced in Intel architectures, such as MMX technology and streaming SIMD extensions (SSE) $[5,6]$. The SIMD technology enables multiple arithmetic or logic operations to be executed simultaneously, so as to improve the efficiency of the program. Thus, we will present programming-level analysis to show the suitability of our approach for general-propose processors with SIMD instructions.

\section{A. The algorithmic-level analysis}

\section{A.1 The proposed approach}

From the previous subsection, we need to analyze the complexity of $\left[S_{N}\right]$. According to Eqn.(14), $\left[M^{\prime}{ }_{N / 2}\right]^{-1}\left[K_{N / 2}\right]^{-1}$ $=\left[\hat{T}_{N / 2}\right]\left[Q_{N / 2}^{\prime}\right]\left[\hat{T}_{N / 2}\right]^{-1}\left[\widetilde{P}_{N / 2}\right]\left[L_{N / 2}\right]^{-1}\left[\widetilde{P}_{N / 2}\right]$ needs to be computed first and then do $N$ more additions for $\left[\begin{array}{cc}{\left[I_{N / 2}\right]} & {\left[I_{N / 2}\right]} \\ {\left[I_{N / 2}\right]} & -\left[I_{N / 2}\right]\end{array}\right]$. It can be shown that

$$
\left[L_{N / 2}\right]^{-1}=\left[\begin{array}{cccccc}
1 & 0 & . & . & \cdot & 0 \\
\frac{1}{2} & \frac{1}{2} & 0 & \cdot & \cdot & 0 \\
0 & \frac{1}{2} & \frac{1}{2} & 0 & \cdot & 0 \\
\cdot & . & \cdot & \cdot & . & . \\
0 & \cdot & \cdot & \frac{1}{2} & \frac{1}{2} & 0 \\
0 & \cdot & . & 0 & \frac{1}{2} & \frac{1}{2}
\end{array}\right],
$$

and only $(N / 2)-1$ additions are required to compute $\left[L_{N / 2}\right]^{-1}$. A fast DCT algorithm, like the one given in [7 or 8] spends $\mu_{N}^{n+C}=\left(\frac{N}{2}\right) \log _{2} N$ multiplications (denoted as $\mu_{*}^{D C r} \mathrm{M}$ ) and $v_{v}^{\alpha \times T}=\left(\frac{3 N}{2}\right) \log _{2} N-N+1$ additions (denoted as $v_{N}^{\alpha * T} \mathrm{~A}$ ) for $\left[T_{N}^{\prime \prime}\right]$

to compute a 1-D $N$-point DCT. Both $\mu_{\left[S_{s]}\right]}$ and $v_{\left[S_{s} \mid\right.}$ are applicable to $\left[\hat{T}_{N}\right]$, because $\left[\hat{T}_{s}\right]$ is obtained by omitting the scale factor and rearranging the rows and columns of $\left[T_{*}^{\prime \prime}\right]$. Hence the required number of multiplications $\mu_{\left[s_{n}\right]}$ and additions $v_{1 s, 1}$ are

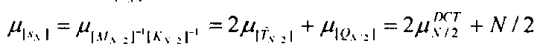

and

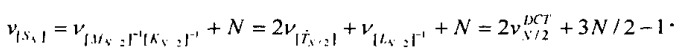

If the scale factors were not absorbed into the quantization stage (the so-called with scale case), we need at most $N$ more multiplications to obtain our result. Thus, the proposed algorithm totally needs

$$
\left(\mu_{N}^{D C T}+N\right) \mathrm{M} \text { and }\left(v_{N}^{D C T}-3 N / 2\right) \mathrm{A}
$$

for splitting an $N$-point DCT block into two (N/2)-point DCT blocks. Splitting an $N \times N$ DCT block into two $(N / 2) \times N$ (or $N \times(N / 2))$ DCT blocks can be realized by performing 1-D split/merge algorithm along each row (or each column), and therefore, our algorithm needs $\left(N\left(\mu_{N}^{\prime x T}+N\right)\right) \mathrm{M}$ and $\left(N\left(v_{N}^{O C T}-3 N / 2\right)\right) \mathrm{A}$ to accomplish this kind of these tasks. Splitting an $N \times N$ DCT into four $(N / 2) \times(N / 2)$ DCTs can be realized by performing $1-D$ split/merge algorithm along each row and then along each column. Our algorithm needs $\left(2 N\left(\mu_{N}^{o c T}+N\right)\right) \mathrm{M}$ and $\left(2 N\left(v_{N}^{O C T}-3 N / 2\right)\right) \mathrm{A}$ in total, for the with scale case.

\section{A.2. Other approaches}

1) Direct approach: The direct approach takes one $N$-DCT, and then takes two forward $(N / 2)$-DCTs for splitting an $N$ DCT block into two $(N / 2)$ DCT blocks. The amount of computation for two dimensional case is $\left(2 N\left(\mu_{N}^{D C T}+2 \mu_{N / 2}^{D C T}\right)\right) \mathrm{M}$ $+\left(2 N\left(v_{N}^{O C C}+2 v_{N / 2}^{O C r}\right)\right)$ A. Similarly, $\left(N\left(\mu_{N}^{O C r}+2 \mu_{N / 2}^{D C r}\right)\right) \mathrm{M}+$ $\left(N\left(v_{N}^{D C T}+2 v_{N / 2}^{D C T}\right)\right) \mathrm{A}$ are needed for computing two $(N / 2) \times N$ (or $N \times(N / 2))$ 2-D DCT blocks.

2) A. N. Skodras's approach: An efficient method for direct computing an $N$-point DCT from two given adjacent N/2-point DCTs has been proposed in [9]. The amount of computations is the same as our proposed approach.

In TABLE I, the numbers of operations needed in different approaches for performing the above DCT splitting tasks are tabulated. By examining the direct approach, the scaling factor had no effect on the multiplication counts in their approach. It is clear that the proposed algorithm is more efficient than the direct one and as well as the A. N. Skodras's approach.

TABLE I

THE NUMBERS OF OPERATIONS REQUIRED FOR SPLITTING AN $N \times N 2$-D DCT INTO FOUR $(N / 2) \times(N / 2) 2$-D DCTS IN DIFFERENT APPROACHES

\begin{tabular}{|c|c|c|}
\hline \multirow{3}{*}{$\begin{array}{c}\text { Different } \\
\text { case }\end{array}$} & \multicolumn{2}{|c|}{$\begin{array}{c}\text { The number of operations needed for splitting an } N \times N \\
\text { 2-D DCT blocks into four }(N / 2) \times(N / 2) 2-D \text { DCT blocks in: }\end{array}$} \\
\cline { 2 - 3 } & $\begin{array}{c}\text { Our proposed / A. N. } \\
\text { Skodras's approach [9] }\end{array}$ & Direct approach \\
\hline \hline \multirow{2}{*}{ with scale } & $\begin{array}{c}\left(2 N\left(\mu_{N}^{D C T}+N\right)\right)^{M} \\
\left(2 N\left(v_{N}^{D C T}-3 N / 2\right)\right)^{A}\end{array}$ & $\begin{array}{c}\left(2 N\left(\mu_{N}^{D C T}+2 \mu_{N / 2}^{D C T}\right)\right)^{M} \\
\left(2 N\left(v_{N}^{D C T}+2 v_{N / 2}^{D C T}\right)\right)^{A}\end{array}$ \\
\hline \multirow{2}{*}{ non-scale } & $\left(2 N \mu_{N}^{D C T}\right) M$ & $\left(2 N\left(\mu_{N}^{D C T}+2 \mu_{N / 2}^{D C T}\right)\right)^{M}$ \\
& $\left(2 N\left(v_{N}^{D C T}-3 N / 2\right)\right) A$ & $\left(2 N\left(v_{N}^{D C T}+2 v_{N / 2}^{D C T}\right)\right)^{A}$ \\
\hline
\end{tabular}

\section{$B$. The programming-level analysis}

Another way to increase performance of a signal processing task is to execute several computations in parallel. Without algorithmic-level optimization, the direct approach can be speeded-up by using today's multimedia instruction set technology. In the following, we will show that our splitter/merger is much more suitable to be realized on the processors, with SIMD instructions, than other approaches.

\section{B.1. SIMD instructions}

Intel Pentium 4 processors extended SIMD computation model with the introduction of SSE2, which operates on packed double (float)-precision data elements as well as 128-bit packed integers. The full set of SIMD capability greatly improves the performance of multimedia related applications. For example, a 128-bit SSE2 register can be 
used to store up to 16 units of 8-bit integers, and up to 16 arithmetic operations can be executed simultaneously by using two SSE2 registers. This results in a significant performance improvement, especially for video encoding.

In our splitter/merger, multiplication of matrices is the key. The SEE2 instructions, PMADDWD and PADDD, can facilitate this implementation. Fig.3 shows an example for computing the first row of the multiplication of two $4 \times 4$ matrices. Matrix $X$ and $Y$ are loaded into SSE registers in a particular form. After that, PMADDWD is performed on these registers in pairs. Eight multiplications are performed on pairs of 16-bit integers simultaneously. And the results, in pairs, are added together and stored in one SSE register as 32-bit integers. Then the two resulting 128-bit data are added together in the 32-bit integer precision by PADDD, hence the first row of the resulting matrix is obtained right way. For the next three rows, similar steps utilized. Therefore, 8 PMADDWDs and 4PADDDs are required for realizing $4 \times 4$ matrix multiplications. The time spent, in this approach, is much less than that of $4^{3}$ multiplications and $3 \times 4^{2}$ additions required in the direct calculation.

$\left[\begin{array}{llll}r_{00} & r_{01} & r_{02} & r_{03} \\ r_{10} & r_{11} & r_{12} & r_{13} \\ r_{20} & r_{21} & r_{22} & r_{23} \\ r_{30} & r_{31} & r_{32} & r_{33}\end{array}\right]=X Y=\left[\begin{array}{llll}x_{006} & x_{01} & x_{02} & x_{03} \\ x_{10} & x_{11} & x_{12} & x_{13} \\ x_{20} & x_{21} & x_{22} & x_{23} \\ x_{301} & x_{31} & x_{32} & x_{33}\end{array}\right]\left[\begin{array}{llll}y_{01} & y_{01} & y_{02} & y_{03} \\ y_{10} & y_{11} & y_{12} & y_{13} \\ y_{20} & y_{21} & y_{22} & y_{23} \\ y_{30} & y_{31} & y_{32} & y_{33}\end{array}\right]$

(a)
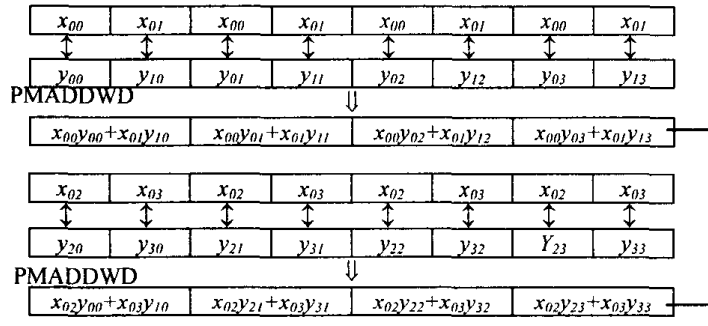

\begin{tabular}{|c|c|c|c|}
\hline 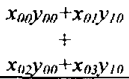 & $\begin{array}{c}x_{n 0} y_{13}+x_{n 1} y_{11} \\
+ \\
x_{02} y_{21}+x_{n 3} y_{31}\end{array}$ & 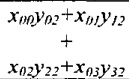 & $\begin{array}{c}x_{00} y_{03}+x_{0 \prime 3} y_{13} \\
+ \\
x_{02} y_{23}+x_{03} y_{33}\end{array}$ \\
\hline \multicolumn{4}{|l|}{ - } \\
\hline$r_{\theta \theta}$ & $r_{n !}$ & $r_{02}$ & $r_{03}$ \\
\hline
\end{tabular}

Fig.3. (a) A $4 \times 4$ matrix-by-matrix multiplication. (b) An example for efficient implementation of $4 \times 4$ matrix-by-matrix multiplication.

\section{B.2. Performance Comparisons}

The proposed splitter/merger and the direct approaches can be speeded-up by SSE2 instructions because both of them are matrix-based algorithms. Let's investigate the most important case of splitting an 8-point DCT into two 4-point DCTs in detailed. The scale factors are saved by absorbing them into the quantization stage in the following analyses.

1) The proposed splitter/merger: We need to count the number of operations required for $\left[S_{8}\right]$. In $(21),\left[\widetilde{P}_{8}\right]$ and $\left[\widetilde{P}_{4}\right]$ can be implemented by shifts and permutations; two PADDDs are required for realizing an $8 \times 1$ vector multiplied by $\left[\begin{array}{ll}{\left[I_{4}\right]} & {\left[I_{4}\right]} \\ {\left[I_{4}\right]} & -\left[I_{4}\right]\end{array}\right]$. 8 PMADDWDs and 4 PADDDs are required for computing the product of $\left[\hat{T}_{4}\right]$ and $\left[\hat{T}_{4}\right]^{-1}$ with a $4 \times 1$ vector, respectively. Since $\left[Q_{4}^{\prime}\right]$ is a diagonal matrix, the matrix-vector product by $\left[Q_{4}^{\prime}\right]$ can be done by one PMADDWD. Since

$$
\left[L_{4}\right]^{-1}=\left[\begin{array}{cccc}
1 & 0 & 0 & 0 \\
\frac{1}{2} & \frac{1}{2} & 0 & 0 \\
0 & \frac{1}{2} & \frac{1}{2} & 0 \\
0 & 0 & \frac{1}{2} & \frac{1}{2}
\end{array}\right]
$$

the matrix-vector product by $\left[L_{4}\right]^{-1}$ can be done by one PADDD and bit shifts. It shows that the total numbers of operations required for the proposed splitter/merger are 17 PMADDWDs and 11 PADDDs.

2) Direct approach: An 8-point IDCT is done first and then two forward 4-point DCTs are taken, which can be written matrix form as

$$
\left[\begin{array}{l}
Y \\
Z
\end{array}\right]=\left[\begin{array}{cc}
{\left[T_{4}\right]} & 0 \\
0 & {\left[T_{4}\right]}
\end{array}\right]\left[T_{8}\right]^{-1}[X]=\left[\begin{array}{cc}
{\left[T_{4} \mid T_{8}\right]_{L, i}^{-1}} & {\left[T_{4} \mathbf{I} T_{8}\right]_{R, i}^{-1}} \\
{\left[T_{4} \mathbb{I} T_{8}\right]_{l, D}^{-1}} & {\left[T_{4} \mathbf{I} T_{8}\right]_{R D}^{-1}}
\end{array}\right][X],
$$

where $\left[T_{8}\right]_{L U}^{-1},\left[T_{8}\right]_{L D}^{-1},\left[T_{8}\right]_{R U}^{-1}$ and $\left[T_{8}\right]_{R D}^{-1}$ are left-up, left-down, right-up and right-down $4 \times 4$ matrices of $\left[T_{8}\right]^{-1}$, respectively. To accomplish Eqn.(23), it needs four $4 \times 4$ matrix-by-matrix multiplications and four $4 \times 4$ matrix-by-vector multiplications. Thus, 40 PMADDWDs and 20 PADDDs are needed.

3) A. N. Skodras's approach: In this the approach, one can not easily load input data into those longer registers, thus this approach can not gain any benefit from SSE2 instructions.

It is shown that the direct approach can optimize its implementation by using SIMD technology, however, without algorithmic-level optimization, the speed-up of the optimization will be limited. Integrating an effective algorithmic-level derivation and programming-level optimizations with SIMD instructions, our approach gets the best performance than other known algorithms.

\section{CONCLUSIONS}

An efficient method for direct splitting of an $N \times N$ 2-D DCT coefficients into four adjacent $(N / 2) \times(N / 2)$, two adjacent $N \times(N / 2)$ (or $(N / 2) \times N)$ 2-D DCT coefficients has been proposed. The algorithmic-level computational complexity of the proposed algorithm is lower than the direct approach and the programming-level analysis tells us that the proposed approach can get the best performance with the aid of available multimedia instruction set technology. Due to its efficiency, the proposed DCT splitter/merger can be applied to realize the transcoding between the latest video coding standard, AVC/H.264, and the older ones, such as MPEG-1, MPEG-2 and MPEG-4 part 2.

\section{REFERENCES}

[1] K. R. Rao and P. Yip, "Discrete Cosine Transform: Algorithms, Advantages and Applications." New York: Academic, 1990.

[2] Hsieh S.Hou, "A Fast Recursive Algorithm For Computing the Discrete Cosine Transform," IEEE Trans. On ASSP, Oct. 1987

[3] ITU-T Rec. H.264 / ISO/IEC FDIS 11496-10, "Advanced Video Coding," Final Draft International Standard, March 2003

[4] ITU-T Rec. H.264 / ISO/IEC I1496-10, "Advanced Video Coding," Final Committee Draft, Document JVT-E022, September 2002

[5] X. Zhou, E. Q. Li, and Y.-K. Chen "Implementation of H.264 Decoder on General-Purpose Processors with Media Instructions," SPIE Conf. on Image and Video Communications and Processing, Jan. 2003.

[6] Y.-K. Chen, N. Yu, and B. Shah, "Digital Signal Processing on MMX Technology," in Programmable Digital Signal Processors: Architecture, Programming and Design, Y. H. Hu, Ed., (Marcel Dekker: NY), pp. 295-331, 2002.

[7] A. N. Skodras, "Fast discrete cosine transform pruning," IEEE Trans.Signal Processing, vol. 42, pp. 1833-1837, July 1994.

[8] C. W. Kok, "Fast algorithm for computing discrete cosine transform," IEEE Trans. Signal Processing, vol. 45, pp. 757-760, Mar. 1997.

[9] Athanassios N. Skodras, "Direct Transform to Transform Computation," IEEE Trans. On SP Letters, Vol.6 No.8. Aug 1999. 